\title{
La elección de las formas de tratamiento en las páginas de tiendas online: Un recorrido por el mundo hispano
}

\author{
Ilpo Kempas (Seinäjoki University of Applied Sciences, Finland)
}

\begin{abstract}
The article deals with the choice of Spanish forms of address on online web stores. It focuses on the choice between informal (tú/vos) and formal address (usted), studied empirically using a corpus created with Google searches. In the context of an online web store, the aim is to build enough confidence in the potential buyer so that they end up buying the product offered even when the seller is not physically present and the customer does not have an opportunity to examine the product. Moreover, payment is done online, which often involves certain risks.

Both tuteo/voseo and ustedeo include elements aimed at building confidence in the potential customer, though from different perspectives. When the customer is addressed with túlvos, the aim is to build confidence including a nuance of familiarity in the sales discourse. By contrast, ustedeo includes nuances of politeness and formality, and conveys the hidden message that since the online web store addresses the customer in a correct and respectful manner, the same good practices also extend to purchase situations.

The article presents results and conclusions related to the following research questions: the general features of the use of forms of address on online web stores; the forms of address on the online web stores in the 21 Spanish-speaking countries of the world; the forms of address in Spanish texts on US online web stores, and pages on which the potential customer is addressed with usted.
\end{abstract}

Keywords: Form of address, online web stores, diatopic variation, tuteo, voseo, ustedeo

\section{Introducción}

En el artículo nos ocuparemos de la elección entre el tratamiento informal (tú/vos) y formal (usted) en las páginas de tiendas online de los países de habla hispana. Este trabajo, basado en un estudio empírico (secciones 3 y 4), constituye una continuación a nuestra investigación sobre este tema (Kempas y Laine 2017). Es un tema interesante porque, tradicionalmente, en español suele tratarse de usted al lector:

(1a) Lea detenidamente las instrucciones de este medicamento.

(1b) Cuando se le solicite, introduzca su nombre de usuario y contraseña.

Por otro lado, el tratamiento informal ha estado en incremento en los registros orales en todo el mundo hispano (NGLE 2010, 1252). Hasta la fecha, no existen estudios que abarquen con precisión todos los países. Los trabajos sobre el tratamiento en el mundo 
hispanohablante (p. ej., Fernández 2006) muchas veces enfocan un aspecto específico, como el voseo. En la sección 2 presentaremos algunos estudios recientes de interés para nuestro estudio.

Este artículo solo investiga el tratamiento en las tiendas online, por lo que el tratamiento en el lenguaje oral en los países examinados cae fuera de su ámbito. Aun así, Kempas y Laine (2017) demuestra una alta correlación entre la forma de cortesía predominante en la sociedad y la forma predominante o exclusiva en las páginas de las tiendas online. Por ello, la bibliografía de la sección 2 nos servirá de punto de comparación para analizar nuestros resultados empíricos.

Se han atestiguado el retroceso del uso de usted a favor de tú en la España posfranquista y las repercusiones de la democratización de la sociedad en el plano de las relaciones interpersonales (ver, p. ej., Sanromán Vilas 2013). Por otro lado, la introducción del tuteo (o del voseo) en Hispanoamérica no puede estar vinculada con España, sino que ha ocurrido independientemente en cada país.

El tratamiento en el lenguaje oral es un problema multifacético que requiere el análisis de un gran número de factores. El punto de partida del presente artículo difiere de la comunicación interpersonal espontánea en varios aspectos: el discurso de venta de las páginas web es escrito, la comunicación es unidireccional y su emisor no se identifica con el nombre. El objetivo de una tienda online es generar la confianza suficiente en el cliente potencial para que este acabe por materializar la compra cuando el vendedor no está físicamente presente y el cliente no tiene la posibilidad de examinar el producto.

La cortesía es un rasgo pragmático muy importante en la comunicación en internet, en general, y en tiendas online, en particular. En inglés se utiliza el término netiquette (net + etiquette) (Yus 2011), en español netiqueta. La elección apropiada entre tratamiento formal e informal puede considerarse como un aspecto importante de la netiqueta. La cortesía excesiva puede contradecir las máximas conversacionales de cantidad y de manera de Grice (1975), porque se espera que la comunicación en la web sea breve y estructuralmente no compleja.

Como género, la lengua de las tiendas online podría caracterizarse como bastante informal, porque el objetivo del texto en las páginas es estimular al lector a actuar. Si la interacción usuario-sitio web es exitosa, el usuario se siente apoderado, involucrado y confidente (Laine 2004).

Tanto el tuteo/voseo como el ustedeo presentan elementos destinados a generar confianza en el cliente potencial, pero desde perspectivas distintas, incluso opuestas. Al tutear/vosear al cliente, el objetivo es generar confianza integrando un toque de familiaridad en el discurso de venta. El ustedeo, por el contrario, presenta matices de cortesía y formalidad e incluye el mensaje disimulado de que, como la tienda online se expresa de manera correcta y respetuosa hacia el cliente, esas buenas prácticas se extienden asimismo a las situaciones de compra.

\section{Las formas de tratamiento en los distintos países hispanohablantes}

Analizaremos a continuación la bibliografía sobre las formas de tratamiento por países relacionada con el tratamiento informal y formal. En este campo, todavía existen muchas lagunas y llama la atención que algunas de las fuentes bibliográficas sean bastante antiguas (p. ej., de los años 1970). Además, muchos de los estudios se limitan a cierto(s) punto(s) geográficos de los países y no son generalizables a los países en cuestión en su conjunto. Aun 
así, las conclusiones presentadas a continuación constituyen una base de comparación útil para nuestros resultados empíricos (sección 4).

Cabe observar que el usted no se emplea exclusivamente como forma de cortesía, respeto y distancia, sino que en algunas variedades se usa además en el tratamiento informal, entre amigos y familiares, inclusive en clases socioculturales baja o medio-baja.

\subsection{América del Sur}

El tratamiento informal se ha generalizado en Argentina, país que en mayor medida que ningún otro se ha perfilado como voseante. Carricaburo escribió ya en 1997 (1997, 24) que los jóvenes voseaban incluso a los adultos que no conocían. Por otro lado, la autora (2010) ha registrado también el uso de usted en contextos de confianza en la zona bonaerense. Kaul de Margalangeon (2010) analiza la elección de formas de tratamiento y la cortesía verbal en cinco folletos de propaganda de servicios argentinos. En tres de ellos, se trata al lector formalmente y en dos de forma informal, respectivamente.

En cuanto a Chile, en la época de publicación de la primera obra de Carricaburo (1997), la autora consideraba el uso de las formas de tratamiento en el país como complejo (ver también Torrejón 1991). Torrejón (2010) concluye que el voseo mixto (tú + voseo verbal) se ha extendido en Chile estas últimas décadas como forma de tratamiento íntimo entre iguales. Por último, Uber (2010, 1057-1063) demuestra la alta frecuencia del tratamiento informal en ámbitos laborales de Chile (Santiago).

En Uruguay, aparecen el tuteo y el voseo (y sus formas híbridas). Según Steffen $(2010,459)$ hay progreso hacia el tratamiento informal, aunque, por otro lado, se ha registrado también una alta frecuencia del usted en distintos ámbitos profesionales (Elizaincín \& Díaz 1981, 85).

Junto con Argentina, Paraguay se perfila como un país voseante (Steffen 2010, 434). Steffen señala que el usted se usa en Paraguay como tratamiento deferencial, pero no dice nada sobre su frecuencia de uso o si la sociedad paraguaya es caracterizada por el trato informal o formal. La razón es la ausencia de trabajos especializados en estos temas (págs. 442-443).

Bolivia se divide en dos zonas en cuanto al uso de las formas de tratamiento (Hummel 2010c, 403). En el este se usan el vos y el usted, mientras que en la zona andina del suroeste aparecen el tú y el vos en el tratamiento informal, con el tuteo verbal. Además, en el este, el usted se utiliza como forma de tratamiento íntimo; en la zona andina, para el tratamiento formal.

En cuanto a Perú, según Hummel (2010b, 396), no existen datos empíricos sobre el reparto entre tú y usted, pero usted sigue teniendo un papel importante como tratamiento estándar, del que se pasa rápidamente al tú en presencia de un marcador de confianza.

En Colombia, según Placencia (2010, 347-248), se usa usted para dirigirse a personas de confianza y para expresar solidaridad, aparte de su valor tradicional de distancia (ver también Uber 1985). Esto concierne sobre todo a Bogotá y puede no ser generalizable a todas las zonas del país. También Bartens (2004) pone de manifiesto el uso de usted de confianza en dicha variedad, aunque demuestra que hay diferencias entre hombres y mujeres.

Placencia $(2010,354)$ señala que en Ecuador el uso del usted se ha registrado en interacciones de servicio en Quito (Placencia 1998) y en Manta (Placencia 2008). 
Venezuela constituye un caso comparable al registrado en Bogotá, Colombia. Según Freites Barros y Zambrano Castro (2010, 904, 917-918), en los Andes de Venezuela usted se emplea tradicionalmente, además de en el tratamiento cortés, entre amigos e iguales. Los autores mencionan (p. 904) el aumento del tuteo en la televisión y radio locales y entre los jóvenes universitarios.

\subsection{Centroamérica}

El uso de usted en el tratamiento informal es un rasgo típico de Costa Rica (p. ej. Lipski 1994, 249). Moser (2010b, 706-707) señala la sustitución de vos por usted en el tratamiento informal, y los años 1970 la época en que probablemente ya existía en San José.

Moser (2010a, 285) concluye que el uso informal de usted se da también en Guatemala, El Salvador y Panamá, aunque no ocurre en la misma escala que en Costa Rica.

Kapovič $(2007,79)$ escribe que en la variedad hondureña se observa una alta preferencia por el usted al hablar a personas desconocidas y en los encuentros callejeros. El tú y el vos se usan para compañeros de trabajo y el voseo es exclusivo entre amigos y en el dominio familiar.

Tocante a México, Vázquez y Orozco $(2010,259)$ mencionan dos estudios de interés. Schwenter (1993) muestra que usted es más frecuente en México en comparación con España, como en gran parte de Hispanoamérica. Reid y Comajoan (2005) concuerdan con Schwenter, pero demuestran que el tuteo se ha generalizado en México durante el lapso de tiempo entre ambos estudios en Ciudad de México y en Guadalajara.

Hummel (2010d) examina el tratamiento en las Antillas hispanohablantes (Cuba, República Dominicana, Puerto Rico). El único pronombre de tratamiento informal allí es tú, por los estrechos vínculos con España (p. 295). No obstante, los países presentan ciertas diferencias en la formalidad del tratamiento. El país más tuteante es Cuba (op. cit., 302; Lipski 1994, 258); quizá por los ideales igualitarios del sistema político. Por el contrario, el ustedeo es común en Puerto Rico: llega, según Rezzi (2003, 124-125), al cien por cien en el trato con desconocidos. En cuanto a la República Dominicana, Hummel (p. 299) menciona el uso de usted en el primer contacto con desconocidos.

En Nicaragua, la mayoría de los informantes de Christiansen $(2014,269)$ opinaban que había que mostrar el respeto y tratar de usted a ciertas personas (familiares mayores o los mayores en general y a algunas personas de cargos elevados).

\subsection{Estados Unidos}

Según Hummel (2010d), la población hispana de Estados Unidos es muy heterogénea y presenta grandes diferencias socioculturales. También las diferencias diatópicas en el origen de la población hispana se reflejan en su actuación lingüística. Según él (p. 488), Nuevo México, con una población hispánica autóctona, presenta rasgos conservadores y favorece el usted para expresar respeto. No obstante, Hummel (loc. cit.) señala asimismo que la tendencia general al tuteo en los países hispanohablantes concierne también a Nuevo México.

Aunque el español del suroeste presenta tendencias hacia el tuteo (Hummel, 490), existen estudios que demuestran la conservación del ustedeo en familias (Brown 1975; Jaramillo 1996) y en relaciones de compadrazgo (Charles de Cerda 1997). Jaramillo (p. 531) opina que la proximidad de México frena la propagación del tuteo en el suroeste. 
Nueva York ha recibido mucha inmigración hispana, sobre todo portorriqueña. Hummel (p. 491-493) se centra en la descripción de los cambios en un grupo de jóvenes inmigrantes (Keller 1974 y 1975), entre los cuales el ustedeo se sustituyó por el tuteo durante el periodo de observación.

\subsection{España}

La España posfranquista se ha caracterizado por la generalización del tuteo. Un buen ejemplo es el comentario de Calderón Campo y Medina Morales $(2010,199)$ que, al comentar sobre el trabajo de Marín (1972), afirman que "en España el avance del tú es mayor que en Hispanoamérica, donde en muchos casos es la alternativa preferida, aunque no la obligada como en España".

En Kempas y Laine $(2017,112)$ demostramos que el tuteo predomina en las páginas de las tiendas online españolas, lo que corresponde a la alta frecuencia del tuteo en la sociedad española. No obstante, el tuteo no es la forma exclusiva, sino que también el ustedeo tradicional está representado, si bien en menor medida. Además, el uso mixto del tuteo y ustedeo en un mismo website es bastante frecuente en las páginas.

\subsection{Guinea Ecuatorial}

Todavía no existen estudios relativos a la elección entre el tratamiento informal y formal en Guinea Ecuatorial. No obstante, Bartens $(2017,18)$ menciona el uso del pronombre usted con la segunda persona del verbo en la variedad en cuestión.

\section{Método y materiales}

Hemos analizado el tratamiento formal e informal en las páginas de las tiendas online de todos los países hispanohablantes. Solo Cuba presentaba algunos problemas, ya que únicamente encontramos una tienda en Cuba; las demás actúan desde el extranjero (Canadá, EE. UU.) y se dirigen a los cubanos que viven en el extranjero, que compran productos para sus familiares. No obstante, es de suponer que esas tiendas extranjeras son muy idóneas para el público receptor.

Los datos se recogieron con Google en septiembre de 2016. Estudiamos primero las páginas de las cuatro tiendas online más importantes de cada país $(n=88)$ o, al menos, de aquellas que salen primero en Google.

Estados Unidos tiene una importante población hispana y algunas tiendas también tienen páginas en español. Nos habíamos dado cuenta anteriormente de que el ustedeo parecía común en las páginas de las tiendas estadounidenses. Por ello, investigamos 47 tiendas online de Estados Unidos que se dirigen al público hispanohablante.

Buscamos en Google también páginas donde al lector se le trata de usted. Los casos $(n=48)$ se recogieron en su orden de aparición. El corpus incluye ocho tiendas no ubicadas en ningún país hispanohablante que se dirigen a clientes de habla hispana por todo el mundo.

\section{Resultados}

Los resultados del análisis geográfico se presentan en la siguiente tabla. 


\begin{tabular}{|c|c|c|c|c|}
\hline País & Tienda 1 & Tienda 2 & Tienda 3 & Tienda 4 \\
\hline Argentina & $\mathrm{V}$ & $\mathrm{V}$ & V & V \\
\hline Chile & $\mathrm{T}$ & $\mathrm{T}$ & $\mathrm{T}$ & $\mathrm{T}$ \\
\hline Uruguay & $\mathrm{T}, \mathrm{V}, \mathrm{U}$ & $\mathrm{U}, \mathrm{T}$ & $\mathrm{T}$ & $\mathrm{V}, \mathrm{T}$ \\
\hline Paraguay & $\mathrm{U}, \mathrm{T}$ & $\mathrm{T}, \mathrm{V}, \mathrm{U}$ & $\mathrm{U}, \mathrm{V}$ & U, V \\
\hline Bolivia & $\mathrm{U}$ & $\mathrm{U}$ & $\mathrm{T}$ & $\mathrm{U}$ \\
\hline Perú & $\mathrm{T}$ & $\mathrm{T}, \mathrm{U}$ & $\mathrm{T}$ & $\mathrm{U}, \mathrm{T}$ \\
\hline Ecuador & $\mathrm{T}$ & $\mathrm{T}$ & $\mathrm{T}$ & $\mathrm{T}$ \\
\hline Colombia & $\mathrm{T}$ & $\mathrm{T}$ & $\mathrm{T}$ & $\mathrm{T}$ \\
\hline Venezuela & $\mathrm{T}$ & $\mathrm{T}$ & $\mathrm{U}, \mathrm{T}$ & $\mathrm{T}$ \\
\hline Guatemala & $\mathrm{T}$ & $\mathrm{T}$ & $\mathrm{U}$ & $\mathrm{T}$ \\
\hline Nicaragua & $\mathrm{T}$ & $\mathrm{T}$ & $\mathrm{T}$ & $\mathrm{U}$ \\
\hline Costa Rica & $\mathrm{T}, \mathrm{U}$ & $\mathrm{V}$ & $\mathrm{T}$ & $\mathrm{T}$ \\
\hline Panamá & $\mathrm{T}$ & $\mathrm{T}$ & $\mathrm{T}$ & $\mathrm{T}, \mathrm{U}$ \\
\hline Honduras & $\mathrm{T}$ & $\mathrm{T}$ & $\mathrm{T}$ & $\mathrm{T}$ \\
\hline El Salvador & $\mathrm{T}$ & $\mathrm{T}$ & $\mathrm{T}$ & $\mathrm{T}$ \\
\hline México & $\mathrm{T}$ & $\mathrm{T}$ & $\mathrm{T}$ & $\mathrm{T}$ \\
\hline Puerto Rico & $\mathrm{T}$ & $\mathrm{T}$ & $\mathrm{U}$ & $\mathrm{T}$ \\
\hline R. Dominicana & $\mathrm{U}, \mathrm{T}$ & $\mathrm{U}, \mathrm{T}$ & $\mathrm{T}, \mathrm{U}$ & $\mathrm{U}, \mathrm{T}$ \\
\hline Cuba & $\mathrm{U}$ & $\mathrm{U}$ & $\mathrm{U}$ & $\mathrm{T}$ \\
\hline Guinea Ec. & $\mathrm{U}$ & $\mathrm{U}$ & $\mathrm{T}, \mathrm{U}$ & $\mathrm{T}, \mathrm{U}$ \\
\hline España & $\mathrm{T}, \mathrm{U}$ & $\mathrm{T}$ & $\mathrm{T}$ & $\mathrm{T}$ \\
\hline
\end{tabular}

Tabla 1: Uso de formas de tratamiento por cuatro de las principales tiendas online de todos los países de habla hispana ( $\mathrm{t}=$ tuteo, $\mathrm{u}=\mathrm{ustedeo}, \mathrm{v}=\mathrm{voseo})$

El tratamiento informal exclusivo aparece en las muestras de Argentina y Chile. Aparece también en la zona ecuatoriana andina, en Ecuador y Colombia. Por último, aparece en Centroamérica, en las tiendas hondureñas, salvadoreñas y mexicanas.

Se aprecia que, por el contrario, el ustedeo exclusivo no aparece en ninguna de las muestras, pero tiene mucho arraigo en Bolivia, Cuba y Guinea Ecuatorial:

(1a) Bienvenido Invitado! ¿Le gustaria entrar? ¿O prefiere crear una cuenta? (tienda boliviana)

(1b) En nuestro supermercado en Internet puede comprar gran variedad de alimentos, electrodomésticos y equipos electrónicos, medicinas, artículos para el hogar y muchos 
otros regalos como cakes, bufets y flores para mandar a $s u$ familia en Cuba. (tienda destinada a los cubanos)

(1c) En cualquier estancia donde esté, usted podrá conectarse a nuestra amplia red Wi-Fi (tienda ecuatoguineana)

El uso mixto del tratamiento informal y formal aparece en más de la quinta parte de los casos $(\mathrm{n}=18)$. En esos, al mismo lector se le trata tanto de tú como de usted en las mismas páginas. El uso mixto es exclusivo en las muestras paraguaya y dominicana.

Como curioso detalle, vemos que en una tienda uruguaya (ejs. 2a, 2b, 2c) y en una paraguaya (ejs. 3a, 3b, 3c) aparecen simultáneamente el ustedeo, el tuteo y el voseo.

(2a) Comprá via web y ahorrá un 18,3\% (voseo)

(2b) Trabaja con nosotros (tuteo)

(2c) Vía Confort, felices de celebrar 50 años junto a usted! (ustedeo)

(3a) Para preguntas referentes a un pedido, más información sobre nuestros productos o porque necesitás contactar con nosotras ¡escribinos! (voseo)

(3b) Hola, escribe un mensaje para iniciar el chat (tuteo)

(3c) Si tiene preguntas o cuestiones sobre esta Política, no dude en contactarnos en cualquier momento (ustedeo)

Los resultados sobre las tiendas estadounidenses se presentan a continuación; el orden de aparición de tú y usted en las dos líneas inferiores se refiere a la predominancia de una u otra forma:

\begin{tabular}{|rr}
\hline usted & $24(51,1 \%)$ \\
\hline tú & $19(40,4 \%)$ \\
\hline tú, usted & $3(6,4 \%)$ \\
\hline usted, tú & $1(2.1 \%)$ \\
\hline Total & $\mathbf{4 7}$
\end{tabular}

Tabla 2: Uso de formas de tratamiento en las páginas de 47 tiendas online estadounidenses

Vemos que el ustedeo exclusivo (51,1\%; ej. 4a) o combinado con el tuteo $(59,6 \%)$ resulta más común que el tuteo exclusivo $(40,4 \%$; ej. $4 b)$.

(4a) Introduzca su dirección de USGoBuy al momento de pagar. No se olvide de poner su número de buzón único en la segunda línea de la dirección de envío.

(4b) Mira las fotos de nuestro desfile de modas de otoño de 2016. ¿Quieres aparecer tú?

Este resultado es interesante porque en la tabla 1 México, el país vecino y el origen de muchos hispanos, se perfila como tuteante. En esa tabla, el tuteo es predominante también en Puerto Rico, pero en las páginas destinadas a los cubanos prevalece el ustedeo. 
Vemos también que las páginas estadounidenses son muy consistentes en el uso de una u otra forma $(91,5 \%)$.

Se nos ocurren dos posibles explicaciones para la frecuencia elevada del ustedeo, que es algo superior a la del tuteo. Es posible que, al usar inglés, en este género se prefiera un mayor grado de formalidad, pese a que el inglés no distingue entre tú y usted en su sintaxis pronominal y verbal.

También puede plantearse que las personas hispanohablantes que han escrito los textos y que son inmigrantes de primera, segunda o tercera generación, no se han visto influenciados por los cambios ocurridos en los países hispanos estas últimas décadas y la generalización del tuteo.

Los resultados de la búsqueda con determinados términos se presentan a continuación:

\begin{tabular}{|lrr|}
\hline País & No de tiendas & Uso mixto de T/V y U \\
\hline España & 23 & 20 \\
\hline Internacional & 8 & 3 \\
\hline México & 3 & 3 \\
\hline EE. UU. & 2 & - \\
\hline Colombia & 2 & 2 \\
\hline Argentina & 2 & 2 \\
\hline Ecuador & 2 & 2 \\
\hline Costa Rica & 2 & 1 \\
\hline Chile & 1 & 1 \\
\hline Panamá & 1 & 1 \\
\hline Venezuela & 1 & - \\
\hline Portugal & 1 & - \\
\hline Total & 48 & $35(72,9 \%)$ \\
\hline
\end{tabular}

Tabla 3: Una mirada sobre el ustedeo: páginas de tiendas online buscadas con los términos de búsqueda tienda, online y pague usted

En primer lugar, observamos que incluso en algunos países que se perfilaban como tuteantes o voseantes (tabla 1) hay tiendas que tratan al cliente de usted. Demuestra que el ustedeo no está del todo excluido y que el tuteo no está tan arraigado como se esperaría.

Por otro lado, en casi las tres cuartas partes el ustedeo aparece junto con el tuteo o voseo (cf. las tablas anteriores).

Muchas veces, el tratamiento informal se emplea en el discurso de venta, como en

(5) Para añadir o cambiar artículos en tu pedido, llámanos al [...].

Por el contrario, el ustedeo es común al especificar los términos de venta, como (en la misma tienda española) en 
(6) Si usted no comprende estos Términos de venta o desea hacer preguntas relacionadas con los mismos, comuníquese con nosotros.

\section{Conclusiones y discusión}

Hemos visto que (tabla 1) el tratamiento informal es exclusivo en las tiendas argentinas y chilenas, así como en las ecuatorianas, colombianas, hondureñas, salvadoreñas y mexicanas. Es de suponer, pues, que en esos países el tuteo se ha extendido al discurso de venta de las tiendas online. Entre los países anteriores, el uso de usted como marcador de confianza y solidaridad se ha registrado en Colombia y El Salvador (sección 2). Podemos excluir, por consiguiente, el uso del usted en la muestra para dirigirse al cliente en el modo "íntimo", integrando un matiz de familiaridad en el discurso de venta.

De la tabla se desprende también el predominio del usted en las tiendas bolivianas, cubanas y ecuatoguineanas, aunque no de forma exclusiva: aparece también el tú. En lo que respecta a Bolivia, el usted tiene dos valores opuestos: en la zona andina se usa para el tratamiento formal, en el este para el tratamiento íntimo (apartado 2.1). Las tres tiendas ustedeantes están ubicadas en la zona andina, en La Paz y en Cochabamba. Por lo tanto, en todos los casos se trata del ustedeo de cortesía y formalidad.

En las tiendas cubanas / destinadas a los cubanos predomina el ustedeo, aunque el tuteo suele considerarse característico de Cuba.

No existen estudios empíricos exhaustivos sobre Guinea Ecuatorial; sin embargo, el uso del pronombre usted con la segunda persona del verbo es común en esa variedad (Bartens 2017), lo que dificulta el análisis; habría que indagar las percepciones de los hablantes de dicha variedad del grado de formalidad del usted en el discurso de venta.

Puede plantearse si la preferencia por el usted en Bolivia, Cuba y Guinea Ecuatorial se explica por la posición aislada de esos países en relación con los demás países. En el caso de Cuba se explicaría por factores políticos, y para los otros dos por factores geográficos. En el caso de Guinea Ecuatorial, el aislamiento geográfico es fácil de comprender, y la geografía de Bolivia, de carácter extremadamente montañoso, puede enlentecer la propagación de innovaciones.

Las muestras paraguaya y uruguaya presentan tres formas de tratamiento, el tuteo, el voseo y el ustedeo (tabla 1). La uruguaya corresponde bien a lo señalado en 2.1 acerca de dicha variedad, pero es interesante que en la paraguaya haya también tuteo, ya que Paraguay se considera como uno de los países en los que el voseo tiene más arraigo (Steffen 2010):

(7) Si deseas tramitar tu pedido tienes la opción de hacerlo registrándote o finalizar la compra sin registro (muestra paraguaya)

La tabla 2 confirma nuestras observaciones previas del ustedeo en las tiendas estadounidenses: supera al tuteo. El ustedeo ha existido tradicionalmente en el lenguaje de la población hispana, si bien la tendencia es hacia el tuteo (apartado 2.3). Puede plantearse si su frecuencia bastante elevada se explica por influencias del inglés o por la falta de contactos con los demás países del mundo hispano, donde el paso del ustedeo al tuteo ha sido más rápido. Silva-Corvalán (1993, 38-39) opina que el inglés favorece o desfavorece estructuras ya existentes en español. Hummel (2010d, 497) piensa que el inglés precisamente contribuiría a la propagación del tú a expensas del usted a través del bilingüismo situacional, en el que las relaciones laborales, que requerirían el usted, se realizan en inglés. Asimismo, al adoptar las 
costumbres de la sociedad moderna urbana, los hispanos también van abandonando sus patrones lingüísticos tradicionales. Por lo tanto, consideramos el conservadurismo linguiístico, la ausencia de contactos con el mundo hispano exterior, como la explicación más plausible para la frecuencia elevada del usted en la tabla 2, aunque cabe tener en cuenta que entre los hispanos ocurre también nivelación dialectal (Otheguy y Zentella 2012). Los futuros estudios podrían extenderse también a otros tipos de documentos online estadounidenses, como a documentos públicos destinados a lectores hispanohablantes.

La tabla 3 revela que el ustedeo tampoco está completamente excluido en países que favorecen el tuteo: 23 de las 48 tiendas son de España, país que marcadamente prefiere el tuteo.

(8) Usted puede pagar con Paypal, Visa, Mastercard, etc. o bien seleccionar la opción de transferencia bancaria, con total seguridad.

Los ejemplos como el anterior contradicen la idea general de que España sería una sociedad casi completamente tuteante.

Un fenómeno muy llamativo en la tabla es el uso mixto del tuteo/voseo y del ustedeo (72,9\% de los casos). Demuestra que la elección de la forma de tratamiento presenta inseguridad en todo el mundo hispánico. Además, es evidente que las tiendas de los países hispanohablantes no consideran el uso mixto del tú y usted como problemático. La explicación natural es que las páginas han sido escritas por varias personas y es difícil coordinar un conjunto compuesto por secciones accesibles a través de enlaces.

Puede plantearse si los resultados obtenidos corresponden al tratamiento en la lengua oral espontánea. El uso de formas de tratamiento en la comunicación interpersonal es un fenómeno multidimensional. Además, depende esencialmente de factores intrínsecos a cada sociedad. No obstante, otro estudio nuestro (Kempas y Laine 2017), en el que tratamos más lenguas, demuestra que la forma de tratamiento de las páginas de las tiendas online presenta una alta correlación con el uso de la forma de cortesía predominante en la sociedad y la forma predominante o exclusiva en las tiendas online.

Por otro lado, consideramos del todo posible que la lengua espontánea oral y la lengua de las tiendas online puedan no ser coincidentes en alguna sociedad. También la tradición de tratar al lector de usted (ejs. 1a y 1b) puede frenar la propagación del tratamiento informal en el género que nos ocupa. Se puede hipotetizar, además, que el contexto anónimo de una tienda online podría, en algunos casos, favorecer el tuteo, ya que el emisor del mensaje no es una persona concreta. También algunos tipos de productos, como los juguetes, pueden tener el mismo efecto.

Hemos presentado un panorama general de las formas de tratamiento en las tiendas online de los distintos países hispanohablantes. La inclusión de más tiendas de los países ofrecería seguramente más detalles de interés. Los futuros estudios podrían relacionarse con el uso de las formas de tratamiento en el discurso de venta audiovisual y su correlación con el discurso en los textos de las tiendas online. Además, el análisis podría extenderse a otros tipos de textos online dirigidos al público; p. ej., para ver si la sustitución del lenguaje persuasivo del discurso de venta por un estilo más neutral refleja la elección de la forma de tratamiento.

Por último, aparte del tratamiento en la variedad ecuatoguineana (apartado 2.5), como tema para un nuevo estudio podría mencionarse la discrepancia entre la idea generalmente 
admitida de que Cuba es tuteante con nuestros resultados, que en las páginas de estas tiendas online predomina el ustedeo.

\section{Bibliografía}

Álvarez Muro, Alexandra, y Francisco Freites Barros. 2010. "Los estudios sobre pronombres de segunda persona en Venezuela." En Formas y fórmulas de tratamiento en el mundo hispano (eds.) Martin Hummel, Bettina Kluge, y María Eugenia Vázquez Laslop, 326339. México: El Colegio de México y Karl-Franzens-Universität Graz.

Bartens, Angela. 2004. "Notas sobre el uso de las formas de tratamiento en el español colombiano actual." En Pronombres de segunda persona y formas de tratamiento en las lenguas de Europa, (eds.) Félix Blanco, y José Amenos. Madrid: Centro Virtual Cervantes-Instituto Cervantes.

https://cvc.cervantes.es/lengua/coloquio_paris/ponencias/pdf/cvc_bartens.pdf

Bartens, Angela. 2017. "Hablar una lengua románica en el África Luso-Hispana: el caso del español ecuatoguineano y del portugués angoleño." En Romance Languages: Multilingualism and Language Acquisition, (eds.) Anna Gudmunson, Laura Álvarez López, y Camilla Bardel, 11-35. Frankfurt am Main: Peter Lang.

Brown, Dolores. 1974. "The use of tú and usted with parents by some Mexican American students." Hispania 58, 1: 126-127.

Calderón Campos, Miguel, y Francisca Medina Morales. 2010. "Historia y situación actual de los pronombres de tratamiento en el español peninsular." En Formas y fórmulas de tratamiento en el mundo hispano (eds.) Martin Hummel, Bettina Kluge, y María Eugenia Vázquez Laslop, 195-222. México: El Colegio de México y Karl-FranzensUniversität Graz.

Carricaburo, Norma. 1997. Las fórmulas de tratamiento en el español actual. Madrid: Arco Libros.

Carricaburo, Norma. 2010. "El ustedeo, un fenómeno que avanza en la Argentina." En Formas y fórmulas de tratamiento en el mundo hispano (eds.) Martin Hummel, Bettina Kluge, y María Eugenia Vázquez Laslop, 888-900. México: El Colegio de México y Karl-Franzens-Universität Graz.

Charles de Cerda, Dora Elia.1997. El uso de tú y usted en el valle del Río Grande. Master of Arts thesis. A \& M, University-Kingsville, Ann Arbor: UMI Dissertation Services.

Christiansen, Ane. 2014. "'El vos es el dialecto que inventamos nosotros, la forma correcta es el tú.' Creencias y actitudes lingüísticas acerca de las formas de tratamiento y la influencia de estas en la educación escolar en Nicaragua." Borealis: An International Journal of Hispanic Linguistics, 2014, 3 / 2: 259-297.

Elizaincín, Adolfo \& Díaz, Olga (1981). "Sobre tuteo / voseo en el español montevideño”, en Adolfo Elizaincín (ed.): Estudios sobre el español de Uruguay. Montevideo: Universidad de la República, 81-86.

Fernández, Mauro. 2006. Pronombres de segunda persona y fórmulas de tratamiento en español: una bibliografía. Lingüística en la red (linred) 22/08/2006. Consulta 25/9/2017. http://www.linred.es/informacion_pdf/informacion13_06072006.pdf 
Freites Barros, Francisco \& Zambrano Castro, Wílmer (2010). "De la preferencia de usted sobre tú en el habla venezolana", en Martin Hummel, Bettina Kluge \& María Eugenia Vázquez Laslop (eds.): Formas y fórmulas de tratamiento en el mundo hispano. México: El Colegio de México y Karl-Franzens-Universität Graz, 902-921.

Grice, Paul H. 1975. "Logic and conversation." En Speech Acts, eds. Peter Cole, y Jerry L. Morgan, 41-58. New York: Academic Press.

Hummel, Martin. 2010a. "El estudio de las formas de tratamiento en las Antillas hispanohablantes." En Formas y fórmulas de tratamiento en el mundo hispano, eds. Martin Hummel, Bettina Kluge, y María Eugenia Vázquez Laslop, 294-323. México: El Colegio de México y Karl-Franzens-Universität Graz.

Hummel, Martin. 2010b. "El estudio de las formas de tratamiento en Perú." En Formas y fórmulas de tratamiento en el mundo hispano, eds. Martin Hummel, Bettina Kluge, y María Eugenia Vázquez Laslop, 376-398. México: El Colegio de México y KarlFranzens-Universität Graz.

Hummel, Martin. 2010c. "Las formas y fórmulas de tratamiento en Bolivia." En Formas y fórmulas de tratamiento en el mundo hispano, eds. Martin Hummel, Bettina Kluge, y María Eugenia Vázquez Laslop, 399-411. México: El Colegio de México y KarlFranzens-Universität Graz.

Hummel, Martin. 2010d. "La investigación de las formas y fórmulas de tratamiento en la diversidad sociolingüística del español en Estados Unidos: una tarea pendiente." En Formas y fórmulas de tratamiento en el mundo hispano eds. Martin Hummel, Bettina Kluge, y María Eugenia Vázquez Laslop, 484-504. México: El Colegio de México y Karl-Franzens-Universität Graz.

Jaramillo, June Aurora. 1996. "Tú and usted: Address etiquette in the Mexican American Family." Hispanic Journal of Behavioral Sciences 18, 4: 522-532.

Kapovič, Marko. 2007. "Fórmulas de tratamiento en dialectos de español; fenómenos de voseo y ustedeo." Hieronymus I (2007): 65-87.

Kaul de Marlangeon, Silvia (2010). "Voseo, ustedeo y cortesía verbal en folletos de propaganda argentinos." En Formas y fórmulas de tratamiento en el mundo hispano, eds. Martin Hummel, Bettina Kluge, y María Eugenia Vázquez Laslop, 993-1011. México: El Colegio de México y Karl-Franzens-Universität Graz.

Keller, Gary D. 1974. "La norma de solidaridad y la de poder en los pronombres de tratamiento: un bosquejo diacrónico y una investigación del español de Nueva York." The Biligual Review / La Revista Bilingüe 1: 42-58.

Keller, Gary D. 1975. "Spanish tú y usted: Patterns of interchange." En Colloquium on Spanish and Portuguese Linguistics 1974, eds. William G. Milan, John J. Staczek, y Juan C. Zamora, 84-96. Washington: Georgetown University Press.

Kempas, y Laine, Päivö. 2017. "Choice of form of address at online web stores: A crosslinguistic and cross-cultural perspective." En Foreign Languages in Changing Times VII: Reviewed Conference Proceedings from an International Scientific Conference, November 11, 2016, eds. Radoslav Štefančík, y Roman Kvapil, 109-114. Bratislava: Vydavatel'stvo EKONÓM.

Laine, Päivö. 2004. Language of interaction in online shopping. Tesis doctoral. Acta Wasaensia No. 123. University of Vaasa. 
Lipski, John M. 1994. El español de América. Madrid: Ediciones Cátedra.

Marín, Diego. 1972. "El uso de tú y usted en el español actual.” Hispania 55:904-908.

Moser, Karolin. 2010a. "Las formas de tratamiento verbales-pronominales en Guatemala, El Salvador, Panamá (y Costa Rica): Hacia una nueva sistematización en la periferia centroamericana." En Formas y fórmulas de tratamiento en el mundo hispano, eds. Martin Hummel, Bettina Kluge, y María Eugenia Vázquez Laslop, 272-291. México: El Colegio de México y Karl-Franzens-Universität Graz.

Moser, Karolin. 2010b. "San José (Costa Rica): Desde los significados pragmáticos del ustedeo en el registro coloquial actual hacia sus primeras manifestaciones en el valle central (Siglo XVIII)." En Formas y fórmulas de tratamiento en el mundo hispano, eds. Martin Hummel, Bettina Kluge, y María Eugenia Vázquez Laslop, 672-713. México: El Colegio de México y Karl-Franzens-Universität Graz.

Nueva gramática de la lengua española (NGLE). 2010. Segunda tirada, corregida. Madrid: Real Academia Española, y Asociación de las Academias de la Lengua Española.Otheguy, Ricardo, y Ana Celia Zentella. 2012. Spanish in New York: Language contact, dialectal leveling, and structural continuity. Oxford: Oxford University Press.

Placencia, María Elena. 1998. "Pragmatic variation: Peninsular vs. Ecuadorian Spanish." Spanish Applied Linguistics 2: 71-106.

Placencia, María Elena. 2008. "Pragmatic variation in corner shop requests in Ecuadorian Andean and Coastal Spanish.” En Variational pragmatics, eds. Klaus Schneider, y Anne Baron, 307-332. Amsterdam: John Benjamins.

Placencia, María Elena. 2010. "El estudio de formas de tratamiento en Colombia y Ecuador." En Formas y fórmulas de tratamiento en el mundo hispano, eds. Martin Hummel, Bettina Kluge, y María Eugenia Vázquez Laslop, 342-373. México: El Colegio de México y Karl-Franzens-Universität Graz.

Reid, Laura, y Comajoan, Llorenç. 2005. "El uso actual de tú y usted en el español peninsular y mexicano." Ponencia presentada en el VII Congreso Nacional de Lingüística, Asociación Mexicana de Lingüística Aplicada \& Universidad de las Américas, 17 al 20 de mayo de 2005, Cholula.

Rezzi, Walter. 2003. Formas de tratamiento en el español de San Juan de Puerto Rico. San Juan: Editorial Plaza Mayor.

Sanromán Vilas, Begoña. 2013. "La elección del tratamiento en el español peninsular." En Représentations des formes d'adresse dans les langues romanes, eds. Elina SuomelaHärmä, Juhani Härmä, y Eva Havu, E., 9-26. Helsinki: Société Néophilologique.

Schwenter, Scott A. 1993. "Diferenciación dialectal por medio de los pronombres: una comparación del uso de tú y usted en España y México." Nueva Revista de Filología Hispánica 41: 127-129.

Silva-Corvalán, Carmen. 1993. "On the permeability of garmmars: Evidence from Spanish and English contact." En Linguistic Perspectives on the Romance languages, eds. William J. Ashby, Marianne Mithun, Giorgio Perissinotto, y Eduardo Raposo, 19-43. Amsterdam - Philadelphia: John Benjamins. 
Steffen, Joachim. 2010. "El tratamiento en Uruguay." En Formas y fórmulas de tratamiento en el mundo hispano, eds. Martin Hummel, Bettina Kluge, y María Eugenia Vázquez Laslop, 449-464. México: El Colegio de México y Karl-Franzens-Universität Graz.

Steffen, Martina. 2010. "El tratamiento en Paraguay." En Formas y fórmulas de tratamiento en el mundo hispano, eds. Martin Hummel, Bettina Kluge, y María Eugenia Vázquez Laslop, 430-448. México: El Colegio de México y Karl-Franzens-Universität Graz.

Torrejón, Alfredo. 1991. "Fórmulas de tratamiento de segunda persona en el español de Chile." Hispania 74: 1067-1076.

Torrejón, Alfredo. 2010. "Nuevas observaciones sobre el voseo en el español de Chile.” En Formas y fórmulas de tratamiento en el mundo hispano, eds. Martin Hummel, Bettina Kluge, y María Eugenia Vázquez Laslop, 755-769. México: El Colegio de México y Karl-Franzens-Universität Graz.

Uber, Diane R. 1985. "The Dual Function of usted: Forms of Address in Bogotá, Colombia." Hispania 68: 388-392.

Uber, Diane R. 2010. "Formas y fórmulas de tratamiento en situaciones laborales en Santiago de Chile y Buenos Aires." En Formas y fórmulas de tratamiento en el mundo hispano, eds. Martin Hummel, Bettina Kluge, y María Eugenia Vázquez Laslop, 1051- 1080. México: El Colegio de México y Karl-Franzens-Universität Graz.

Vázquez Laslop, María Eugenia, y Orozco, Leonor. 2010. "Formas de tratamiento del español en México." En Formas y fórmulas de tratamiento en el mundo hispano, eds. Martin Hummel, Bettina Kluge, y María Eugenia Vázquez Laslop, 247-269. México: El Colegio de México y Karl-Franzens-Universität Graz.

Yus, Francisco. 2011. Cyberpragmatics: Internet-mediated communication in context. Amsterdam/Philadelphia: John Benjamins Publishing Company. 